\section{KEYY
LOCKO \\ Working Together For Stem Cell Research Innovation}

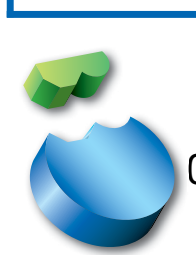

Our Animal Free (AF) enzymes have been discovering facts about life for decades.

That's why Worthington, the primary enzyme producer, has more citations in respected journals like Nature

Methods and rank higher in search engines like $B i o z^{\odot}$.

\section{AF COLLAGENASES $99 \%$ Bioz Rating \\ AF DNASES \\ 92\% Bioz Rating \\ AF STEMxyme ${ }^{\circledast}$ 95\% Bioz Rating}

Unlock the power of Worthington with our NEW Stem Cell

Research Products catalog.

Go to: Worthington-Biochem.com

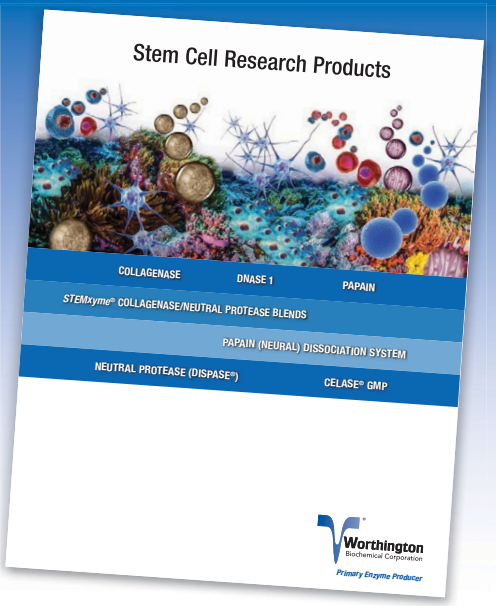

BIOMATERIALS

\title{
Printing in vivo
}

Intravital three-dimensional bioprinting enables the biofabrication of constructs in a pre-existing 3D matrix, such as inside tissues of live animals.

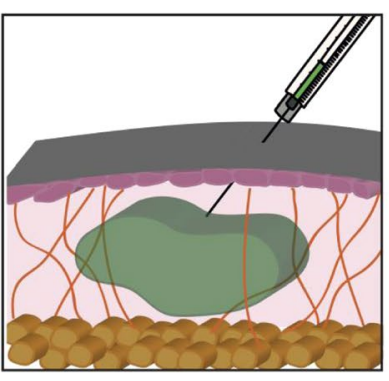

Injection into the tissue

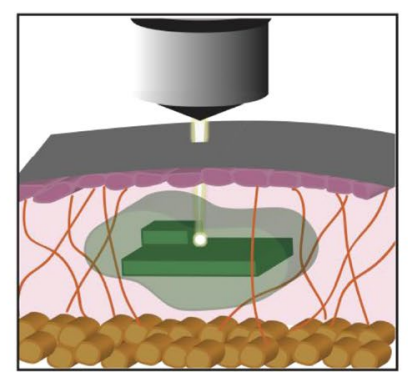

3D bioprinting through the tissue

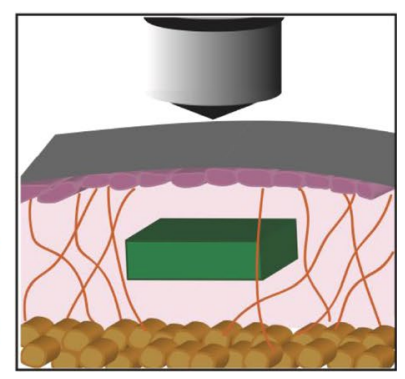

Intravital imaging

The workflow of intravital 3D bioprinting. Reprinted with permission from A. Urciuolo et al. Nat. Biomed. Eng. https://doi.org/10.1038/s41551-020-0568-z (2020), Springer Nature.

$\mathrm{T}$ hree-dimensional (3D) bioprinting has demonstrated its remarkable capability to fabricate complex structures that enables one to integrate cells and synthetic hydrogels into a tissue-like unit. Such engineered structures provide an artificial environment for cell self-organization and cell development. For example, 3D bioprinting has been employed to print organoids as biological models, or customized tissue scaffolds for implantable constructs. Yet it remains challenging to bioprint within a living system.

Nicola Elvassore from the University of Padova, Italy, proposed the idea of bioprinting in a live animal. Elvassore says, "We want to do printing directly into the patient and use the potential of stem cells to make or regenerate tissue into the patient directly." Elvassore and colleagues developed a technique called intravital 3D (i3D) bioprinting, in which a cell-laden polymer solution is injected into the desired location and exposed to near-infrared laser light for solidification. The solidification process is enabled by cross-linking photoactive polymers within the focal voxel, and the spatial position of cross-linking is defined by the laser-scanning path within the $x-y$ plane. The $3 \mathrm{D}$ bioprinted structure is thus accomplished by layer-by-layer addition of the $x-y$ regions.

The first requirement of $\mathrm{i} 3 \mathrm{D}$ bioprinting is real-time imaging. The printed construct needs to be accurately placed at a specific position with a correct orientation. "So it has to be coupled with an intravital imaging," says Elvassore. Second, "the bioprinting through the tissues using the infrared light has to be very efficient." To achieve this, Elvassore and colleagues identified coumarin derivatives that can crosslink synthetic and gelatin polymers quickly via a cycloaddition reaction upon light excitation.

The researchers first tested $\mathrm{i} 3 \mathrm{D}$ bioprinting of a gelatin construct in a pre-existing 3D Matrigel and demonstrated that the i3D printed constructs support the growth of human small intestinal organoids and help to instruct cell organizations. They also demonstrated that a cell-laden gelatin hydrogel can be printed in the dermis and skeletal muscles of mice.

From a biomaterials perspective, the mechanical properties of printed constructs are critical for tissue regeneration. In i3D bioprinting, the hydrogel stiffness can be adjusted by fine-tuning the degree of photo-cross-linking by manipulating the laser power.

Looking forward, Elvassore hopes that the bioprinting system can become more versatile and dynamic so that needed molecules can be delivered in the proper way at the proper time, taking into account the evolution of printed objects (for example, organoids). "We envision that this technology is not limited to specific time, but can do printing successfully, even later on."

\section{Lei Tang}

Published online: 31 July 2020

https://doi.org/10.1038/s41592-020-0920-y

Research paper

Urciuolo, S. et al. Intravital three-dimensional bioprinting. Nat. Biomed. Eng. https://doi.org/10.1038/ s41551-020-0568-Z (2020). 